\title{
Large Scale Synthesis of Superparamagnetic Face-centered Cubic Co/C Nanocapsules by a Facile Hydrothermal Method and their Microwave Absorbing Properties
}

\author{
Xianguo Liu ${ }^{a *}$, Niandu Wu ${ }^{a, b}$, Pingping Zhou ${ }^{a}$, Nannan Bi, ${ }^{a, b}$, \\ Siu Wing Or ${ }^{b}$, Caiyun Cui ${ }^{a}$, Yuping Sun ${ }^{c}$ \\ ${ }^{a}$ Anhui Key Laboratory of Metal Materials and Processing, School of Materials Science and \\ Engineering, Anhui University of Technology, Maanshan 243002, PR China \\ ${ }^{b}$ Department of Electrical Engineering, The Hong Kong Polytechnic University, \\ Hung Hom, Kowloon, Hong Kong \\ ${ }^{c}$ Center for Engineering Practice and Innovation Education, Anhui University of Technology, \\ Maanshan 243032, PR China
}

Received: April 7, 2015; Revised: June 4, 2015

\begin{abstract}
A large scale direct hydrothermal method has been used to prepare face-centered cubic (FCC) $\mathrm{Co} / \mathrm{C}$ nanocapsules with a core of FCC-Co nanoparticles and a shell of amorphous $\mathrm{C}$. The size distribution is $5-20 \mathrm{~nm}$. The $T_{B}$ of FCC-Co/C nanocapsules is determined as $85 \mathrm{~K}$, indicating that $\mathrm{FCC}-\mathrm{Co} / \mathrm{C}$ nanocapsules are superparamagnetic at $300 \mathrm{~K}$. For FCC-Co/C nanocapsules-paraffin composite, an optimal reflection losbs (RL) of $-33.4 \mathrm{~dB}$ is observed at $9.6 \mathrm{GHz}$ for the $2.6 \mathrm{~mm}$ thick layer. RL values exceeding $-20 \mathrm{~dB}$ in the $7.4-15 \mathrm{GHz}$ range are obtained by choosing an appropriate absorption-layer thickness between 1.7 and $3.3 \mathrm{~mm}$. The good microwave absorbing properties of FCC-Co/C nanocapsules is considered to result from the excellent synergetic effect of the multi-dielectric relaxation loss and the multi-magnetic resonance loss. Quantitative calculation demonstrated that not only RL peak position but also the number of the peaks are determined by the quarter-wavelength cancellation model.
\end{abstract}

Keywords: nanocomposites, superparamagnetism, microwave absorbing material

\section{Introduction}

With fast advancement of wireless technology in microwave frequency range, microwave absorbing materials (MAMs) are becoming increasingly important for applications outside special fields such as silent rooms, radar systems, and military application ${ }^{1-5}$. MAMs are generally classified into three types: the resistive loss type, the dielectric loss type and magnetic loss type ${ }^{6}$. Single loss type MAMs limits their application in $\mathrm{GHz}$ frequencies due to their intrinsic disadvantages. The microwave absorbing characteristics could be effectively evaluated by the relatively complex permittivity $\left(\varepsilon_{r}=\varepsilon^{\prime}-j \varepsilon^{\prime \prime}\right)$ and permeability $\left(\mu_{r}=\mu^{\prime}-j \mu^{\prime \prime}\right)$, in which the real and imaginary parts represent the storage and loss of microwave energy in absorbent through various magnetic and/or dielectric phenomena, respectively ${ }^{4}$. Composite materials allow convenient use on surface, good control over mechanical properties, and variation of microwave absorbing properties with proper selection of matrix material and different inclusions, either dielectric, conductive, or ferromagnetic ${ }^{1}$. Low density requirements for the absorber make the nanocomposites the focus of MAMs. Core-shell structured nanocapsules are a special type of nanocomposites, which are usually composed of cores and shells of nanometer size that are made of different materials ${ }^{5}$. Recently, much research has been focused on nanocapsules (dielectric shells and magnetic nanoparticles as cores) as

*e-mail: liuxianguohugh@gmail.com
MAMs. The core-shell structure improves the impedance matching between the nanostructures and the incident microwaves due to the coupling of the electromagnetic field with both the magnetic core and the dielectric shell. Some nanocapsules of this type, including $\mathrm{FeNiMo} / \mathrm{C}, \mathrm{Fe} / \mathrm{ZnO}$, $\mathrm{FeCo} / \mathrm{Al}_{2} \mathrm{O}_{3}, \mathrm{Ni} / \mathrm{Cu}$ oxides, etc., have been studied ${ }^{7-10}$.

The ferromagnetic resonance and dielectric resonance of nanocomposite, essential for enhanced microwave absorption, is determined by a few physical parameters of the nanocomposites, namely, anisotropy coefficient $K$, damping parameter $\alpha$, saturation magnetization $M_{S}$, and particle shape ${ }^{1}$. When nanocomposites exhibit superparamagnetism, the susceptibility and its resonance frequency depend also on volume of magnetic nanoparticles. In addition, with diminishing diameter of nanoparticles the surface effects become increasingly important, affecting primarily the anisotropy coefficient and damping parameter ${ }^{1}$. In our previous work, we synthesized the superparamagnetic graphite-coated $\mathrm{FeNi}_{3}$ nanocapsules by arc discharge method. Compared with ferromagnetic $\mathrm{FeNi}_{3}$ nanoparticles, the natural resonance and attenuation properties of the superparamagnetic graphite-coated $\mathrm{FeNi}_{3}$ nanocapsules were dramatically enhanced ${ }^{11}$. In this paper, we will report the large scale synthesis of superparamagnetic face-centered cubic (FCC) $\mathrm{Co} / \mathrm{C}$ nanocapsules by a facile hydrothermal method. The phase, microstructure, magnetic properties and microwave absorbing properties will be deeply investigated. 


\section{Experimental}

\subsection{Materials}

All the reactions, $\mathrm{CoCl}_{2} \cdot 6 \mathrm{H}_{2} \mathrm{O}, \mathrm{NaOH}$, hydrazine hydrate $\left(\mathrm{N}_{2} \mathrm{H}_{4} \cdot \mathrm{H}_{2} \mathrm{O}\right)$ and glucose, were of reagent grade and used without further purification.

\subsection{Preparation of FCC-Co/C nanocapsules}

In a typical experiment, a solution was first prepared by dissolving $0.1 \mathrm{~g}$ glucose, $4.76 \mathrm{~g} \mathrm{CoCl}_{2} \cdot 6 \mathrm{H}_{2} \mathrm{O}$ and $0.4 \mathrm{~g} \mathrm{NaOH}$ in $40 \mathrm{~mL}$ distilled water. The mixture was stirred vigorously until it was homogeneous and then a $5 \mathrm{~mL}$ volume of $\mathrm{N}_{2} \mathrm{H}_{4} \cdot \mathrm{H}_{2} \mathrm{O}$ was added to the solution. Subsequently, the solution was transferred into a 50-mL autoclave. The autoclave was sealed and put into a furnace, which was preheated to $120^{\circ} \mathrm{C}$. After heating for $12 \mathrm{~h}$, the autoclave was taken out and cooled naturally to room temperature. The product was washed with distilled water and ethanol several times to remove impurities before the characterization.

\subsection{Characterization and measurements}

The phase analysis for the product was performed by using powder X-ray diffraction (XRD), acquired by a Bruker D8 Advance X-ray diffractometer equipped with a monochromatized $\mathrm{Cu}-\mathrm{K} \alpha$ radiation. Raman spectra were studied on a DXR Raman spectrophotometer (Thermo Scientific, USA) that was equipped with a $633 \mathrm{~nm}$ laser source. The morphology and size distribution of the products were observed by a high-resolution transmission electron microscope (HRTEM) images from JEOL-2100F. The magnetic properties were measured by a superconducting quantum interference device (SQUID, Quantum Design MPMS XL-7).

The FCC-Co/C nanocapsules-paraffin composite was prepared by uniformly mixing FCC-Co/C nanocapsules with paraffin, and the detailed descriptions can be found elsewhere ${ }^{7-11}$. The mixture was compressed into a cylinder-shaped compact, and then cut into a toroidal shape with $7.00 \mathrm{~mm}$ outer diameter and $3.04 \mathrm{~mm}$ inner diameter. The EM parameters of FCC-Co/C nanocapsules (40 wt.\%)-paraffin composites were measured using an Agilent N5244A vector network analyzer (VNA, USA). Coaxial method is used to determine the EM parameters of the toroidal samples in the frequency range of 2-18 GHz in transverse EM mode. The vector network analyzer was calibrated for the full two-port measurement of reflection and transmission at each port. The complex permittivity $\left(\varepsilon_{r}=\varepsilon^{\prime}-j \varepsilon^{\prime \prime}\right)$ and complex permeability $\left(\mu_{r}=\mu^{\prime}-j \mu^{\prime \prime}\right)$ were calculated from S-parameters using a simulation program of Reflection/Transmission Nicolson-Ross model ${ }^{10}$.

\section{Results and Discussion}

\subsection{Charaterization of FCC-Co/C nanocapsules}

The XRD pattern in Figure 1 can be clearly shown and indexed to the FCC structure of Co with a lattice parameter of $a=3.544 \AA$ (JCPDS card No. 15-0806), which is close to that reported in the previous literature ${ }^{12,13}$. No other impurity peaks are detected, suggesting the purities of FCC-Co. The strong and sharp peaks reveal that the FCC-Co nanoparticles are well crystallized. The average grain size of FCC-Co is estimated to be $12.7 \mathrm{~nm}$ by using the reflection peak of (111) and Debye-Scherrer's relation. It has been well known that bulk Co has a hexagonal close-packed (HCP) structure at room temperature and undergoes a reverse martensitic phase transition to a $\mathrm{FCC}$ structure at about $415^{\circ} \mathrm{C}$. The existence of the high-temperature phase in nanocapsules at room temperature can be attributed to the higher surface energy of nanoscale particles ${ }^{13}$. In addition, there are no detectable peaks for carbon in the XRD pattern, indicating the amorphous state of the $\mathrm{C}$ shells in the composite. Furthermore, since $\mathrm{C}$ is on the shell of the nanocapsules, it is also difficulty to detect its XRD pattern because of breaking down of the periodic boundary condition (translation symmetry) along radial direction ${ }^{7}$. The inset of Figure 1 shows the Raman spectrum of the products, which can provide more detailed information about the structure of the carbon ${ }^{14}$. It is obviously seen that there are two peaks in the spectrum. The peak at $1347.2 \mathrm{~cm}^{-1}$ is called as D-line, which is from the defects in the graphitic layer, which the peak at $1579.2 \mathrm{~cm}^{-1}$ is identified as G-line caused by the $\mathrm{E}_{2 \mathrm{~g}}$-mode of graphite. The ratio of $I_{D} / I_{G}$ can represent the quality or crystallinity of the carbon in the nanocomposites. The $I_{D} / I_{G}$ ratio exceeds 1 , revealing the amorphous nature of $\mathrm{C}$ in the present nanocomposites ${ }^{14}$.

The morphology and size distribution of the products can be observed clearly in Figure 2a. The particles are of irregular spherical shape, which in a certain sense reveals the crystallization habits of FCC-Co. The morphology of FCC-Co/C nanocapsules is similar with the results reported in Ma et al. ${ }^{12}$ and . Liu et al. ${ }^{13}$. It is understood that the lower-state for the crystallization process dominates the concrete morphology of the $\mathrm{FCC}-\mathrm{Co} / \mathrm{C}$ nanocapsules. As measured from TEM

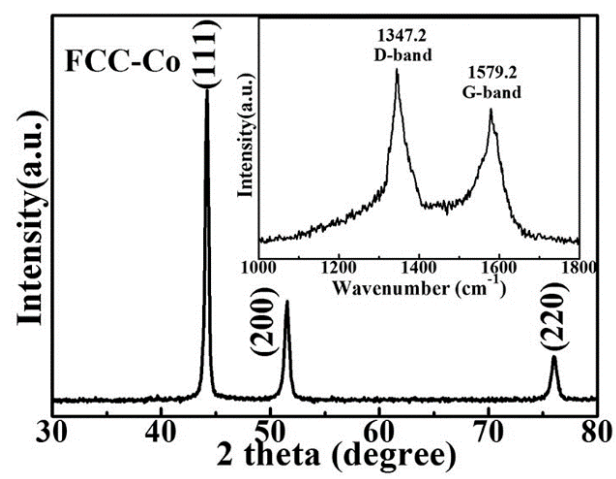

Figure 1. XRD pattern of the products. The inset shows the Raman spectrum of the products.

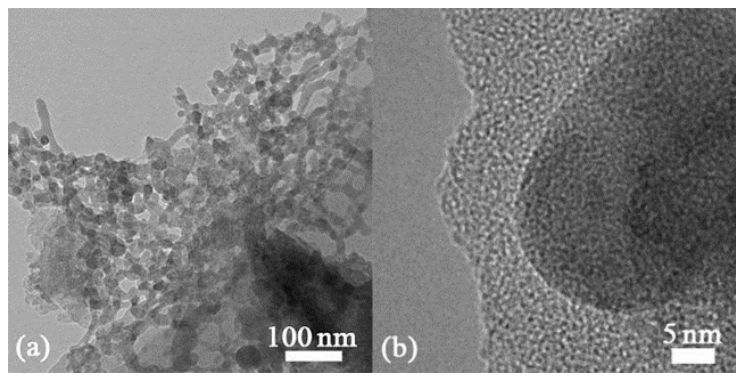

Figure 2. (a) TEM and (b) HRTEM images of the products. 
image, the diameter distribution of nanocapsules ranges from 5 to $20 \mathrm{~nm}$. The averaged diameter is about $13.2 \mathrm{~nm}$, after averaging that of more than 100 nanoparticles, which is onsistent with $12.7 \mathrm{~nm}$ calculated from the XRD (111) peak according to the Scherrer equation. The typical core-shell structure is shown in HRTEM image (Figure 2b), and the shell regions contrast with typical amorphous materials. The amorphous shells are considered as carbon detected by the energy dispersive spectroscopy spectrum. The product is identified to be FCC-Co/C nanocapsules on basis of the results of XRD and TEM.

\subsection{Magnetic properties of FCC-Co/C nanocapsules}

Figure $3 \mathrm{a}$ shows the temperature dependence of the magnetization at an applied field $(H=200 \mathrm{Oe})$ after different cooling process (at 10-300 K) for as-prepared nanocapsules. In the zero-field cooling (ZFC) process, the nanocapsules was cooled from room temperature to $10 \mathrm{~K}$ without application of an external magnetic field, then the magnetization as a function of temperature was recorded at the applied field ( $H=200 \mathrm{Oe})$ during the warming process. In the case of field cooling (FC), the nanocapsules was cooled from 300 to $10 \mathrm{~K}$ in the presence of the external magnetic field ( $H=200 \mathrm{Oe})$, and the magnetization as a function of temperature was recorded with decreasing temperature ${ }^{12}$. The blocking temperature $T_{B}$ of FCC-Co/C nanocapsules is determined as $85 \mathrm{~K}$ from ZFC curve in Figure 3a. The blocking temperature is defined as the temperature above which one particle has enough relaxation time, within the observation time, to reverse its moments to the orientation of the applied field ${ }^{15}$. Namely, $T_{B}$ is the temperature corresponding to the maximum of magnetization in the ZFC curve, indicating a blocking process of the nanoparticles. Below $T_{B}$, the sharp increase of ZFC magnetization can be explained by the contribution of some small particles with the blocking temperature less than $85 \mathrm{~K}$. When the temperature tends to $T_{B}$ other bigger single-domain particles can relax their moments to the applied field within the observation time $^{12}$. From 85 to $300 \mathrm{~K}$, magnetization decreases gradually at thermal agitation effects. Thus the particles show the superparamagnetism. The wide peak around $T_{B}$ in $\mathrm{ZFC}$ curve indicates the wide size distribution of the FCC-Co/C nanocapsules. For the FCC-Co/C nanocapsules, $M_{Z F C}$ and $M_{F C}$ clearly show irreversibility persists up to $\sim 300 \mathrm{~K}$, named by $T_{I R R}$, at which $M_{Z F C}$ and $M_{F C}$ finally coincide ${ }^{16-18}$. For an ideal superparamagnetic material, $T_{B}$ and $T_{I R R}$ are identical so that their difference provides a good indicator of the actual particle size distribution. For our samples, the $T_{I R R}-T_{B}$ differences indicate that some larger nanoparticles remain blocked till temperature is sufficiently high to overcome their energy barriers ${ }^{16-18}$. Figure $3 b$ represents the hysteresis loops recorded at 50 and $300 \mathrm{~K}$, respectively, for $\mathrm{FCC}-\mathrm{Co} / \mathrm{C}$ nanocapsules. The shape of the hysteresis loops is similar, and the $M_{S}$ and the coercive force increase with decreasing the temperature. The occurrence of the hysteresis at $50 \mathrm{~K}$ can be explained to be due to that a slow relaxation process causes the anisotropy energy barriers dominate the rotation of the magnetic moment, and the system cannot respond immediately to the field change to reach its thermodynamic equilibrium state. No coercive force exists in the hysteresis loop at $300 \mathrm{~K}$, further indicating the $\mathrm{FCC}-\mathrm{Co} / \mathrm{C}$ nanocapsules are superparamagnetic. The $M_{S}$ of FCC-Co/C nanocapsules at $300 \mathrm{~K}$ is $70.4 \mathrm{emu} / \mathrm{g}$, which is smaller than the highest values reported for the bulk cobalt $(162 \mathrm{emu} / \mathrm{g})$ and the $133.29 \mathrm{emu} / \mathrm{g}$ of HCC-Co particles ${ }^{19,20}$. This difference in the bulk property is explained by weakly coupled and more disordered spins on the surface and the existence of a nonmagnetic $\mathrm{C}$ component ${ }^{5,11}$.

\subsection{Microwave absorbing properties of $\mathrm{FCC}-\mathrm{Co} / \mathrm{C}$ nanocapsules}

The complex permittivity and complex permeability versus frequency for the $\mathrm{FCC}-\mathrm{Co} / \mathrm{C}$-paraffin composite are shown in Figure $4 \mathrm{a}$ and b. Both the real part $\left(\varepsilon^{\prime}\right)$ and the imaginary part $\left(\varepsilon^{\prime \prime}\right)$ of the complex permittivity in Figure 4a display the similar tendency of decreasing with the frequency increasing from 2 to $18 \mathrm{GHz}$ and exhibit three resonant peaks at around 3.6, 9.6 and $14.8 \mathrm{GHz}$, respectively. One peak may result from the relaxation loss of permanent electric dipoles due to defects in the amorphous carbon shell demonstrated by the TEM image in Figure 2b. The other peaks can be explained on the basis of space charge polarization model of Wagner ${ }^{21}$ and Maxwell ${ }^{20}$ According to space charge polarization model, the increase of $\varepsilon^{\prime}$ of complex permittivity at higher frequencies can be attributed to the interfacial space-charge polarization which arises from the heterogeneous mixtures. Interfacial polarization is always present in heterogeneous mixtures including more than one phase like the FCC-Co/C-paraffin composites. This kind of

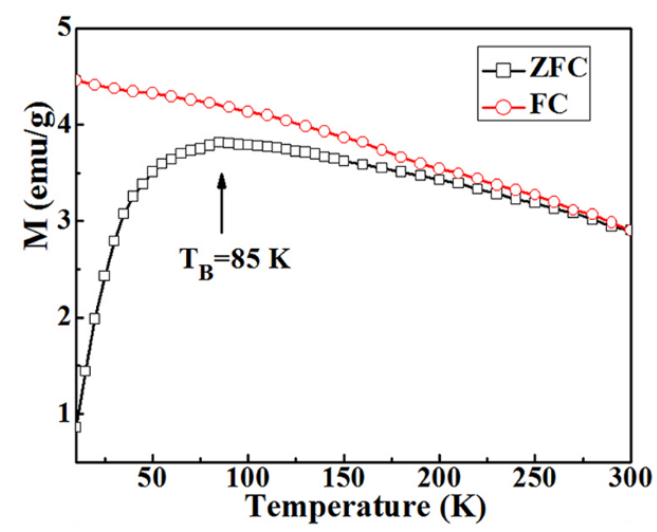

(a)

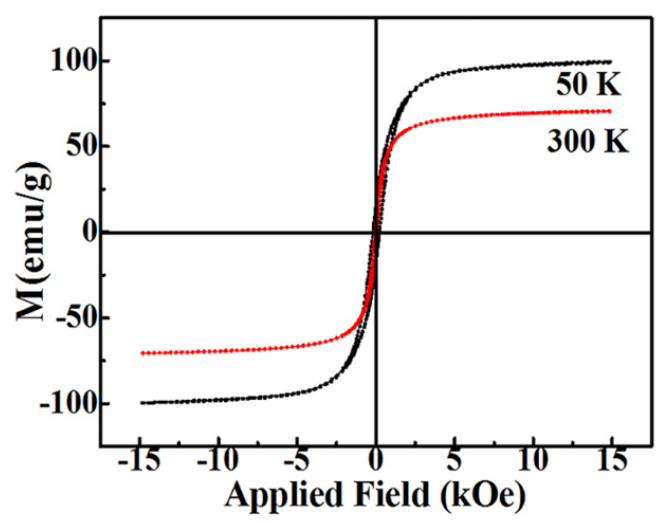

(b)

Figure 3. (a) $\mathrm{ZFC}$ and $\mathrm{FC}(\mathrm{H}=200 \mathrm{Oe})$ magnetization curves, and (b) hysteresis loops at 50 and $300 \mathrm{~K}$ of FCC-Co/C nanocapsules. 


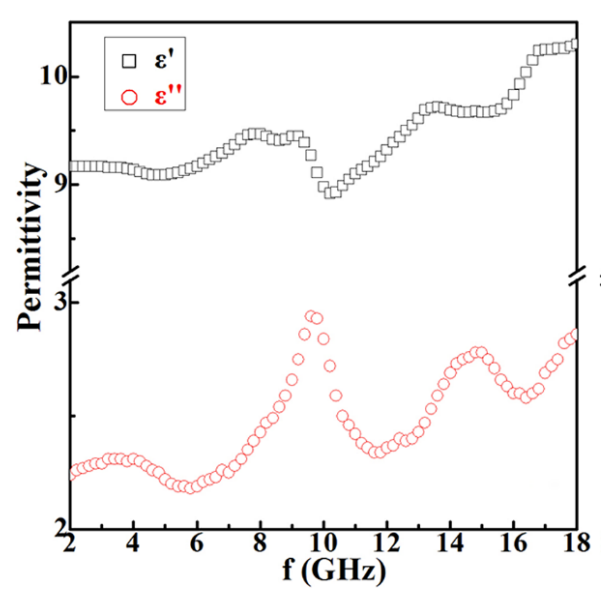

(a)

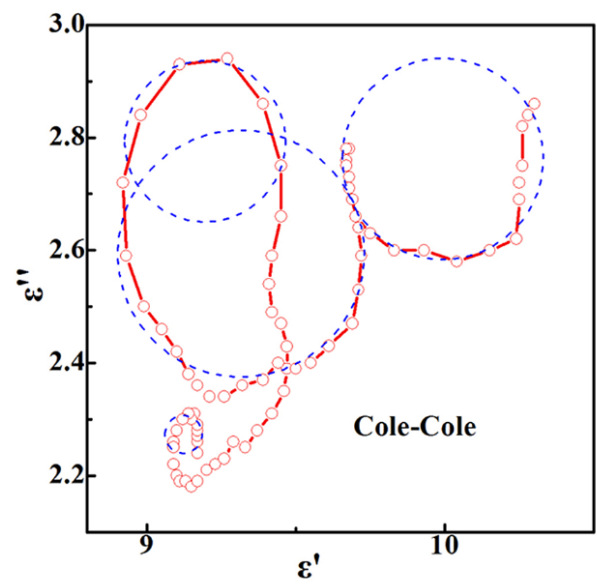

(c)

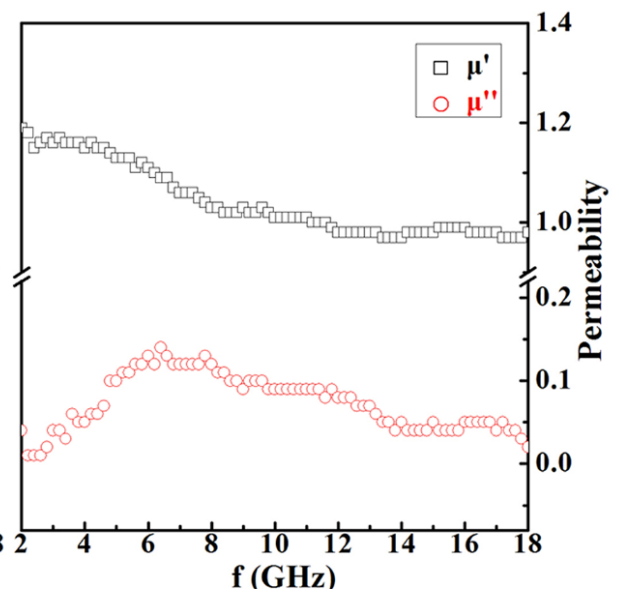

(b)

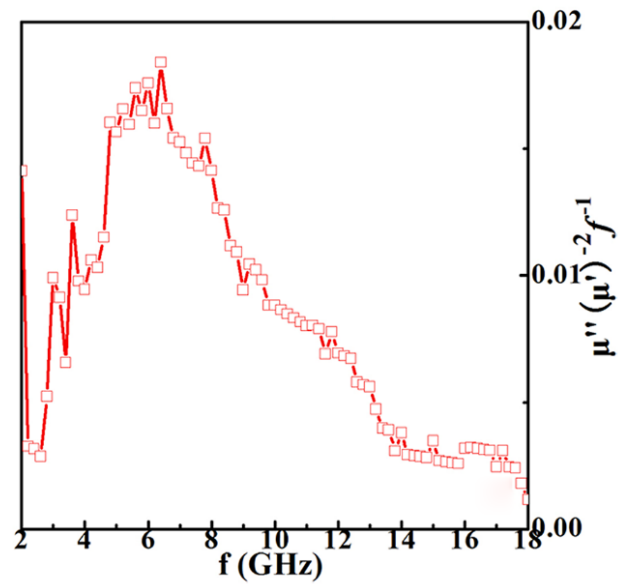

(d)

Figure 4. Frequency dependence of (a) the relative complex permittivity and (b) the relative complex permeability, (c) Cole-Cole plot. The four dashed circles are guides to the eyes, and (d) frequency dependence of $\mu^{\prime \prime}\left(\mu^{\prime}\right)^{-2} f^{-1}$.

polarization arising at the interfaces is due to the migration of charge carriers through different phases of the composite material, which may be attributed to the electronegativity difference between the components. When an external electric filed is applied, the space charge can be appeared, due to the fact that the motion of charges will be hindered at various points of the components material. The appearance of such space charge can distort the macroscopic field and appears as polarization to an external observer ${ }^{20}$. Interfacial polarization is present in materials with considerable electrically heterogeneous. Hence, Composite materials will exhibit large interfacial polarization within them under an external electric field. FCC-Co nanoparticles embedded in an amorphous carbon shells can act as charge centers and can contribute to the enhancement of dielectric permittivity because of interfacial polarization ${ }^{22}$. According to the Debye relaxation theory ${ }^{23}$, for most dynamic processes of dielectric relaxation loss, $\varepsilon^{\prime}$ and $\varepsilon$ " follow the equation of the Cole-Cole semicircle. Clear segments of the Cole-Cole semicircle between $\varepsilon$ 'and $\varepsilon$ " for the present $\mathrm{FCC}-\mathrm{Co} / \mathrm{C}$ nanocapsules are displayed in Figure $4 \mathrm{c}$, in which the multi-semicircles indicate the multi-dielectric relaxation loss.
The frequency dependencies of the real part $\left(\mu^{\prime}\right)$ and the imaginary part $\left(\mu^{\prime \prime}\right)$ of $\mu_{r}$ are presented in Figure $4 \mathrm{~b}$. The value of $\mu$ 'decreases from 1.19 to 0.98 with increasing frequency, which shows excellent frequency dispersion. The $\mu^{\prime \prime}$ has a resonance peak at $6.4 \mathrm{GHz}$, and the large resonance band is observed in the range of 4-14 GHz, which is corresponding to the multi-magnetic resonance, like in $\mathrm{CoNi} / \mathrm{C}$ nanocapsules, $\mathrm{HCP}-\mathrm{Co}$ nanoparticles and Co nanoflakes ${ }^{20,23,24}$. In our previous papers, the resonance frequency at $6.4 \mathrm{GHz}$ is due to the large anisotropy energy. The anisotropy energy of particles of small size, especially in nanometer scale, may be remarkably increased due to the shape/surface anisotropy affected by the size effects ${ }^{5,8-11}$. The large resonance band may be interpreted as a consequence of size and morphology of the FCC-Co/C nanocapsules. As a typical magnetic material, the magnetic loss of FCC-Co/C nanocapsules is mostly associated with magnetic hysteresis, domain wall resonance, eddy current loss, natural resonance, and exchange resonance for particles smaller than $100 \mathrm{~nm}^{[25]}$. Because FCC-Co/C nanocapsules are superparamagnetic and the averaged size of $\mathrm{FCC}-\mathrm{Co} / \mathrm{C}$ nanocapsules is smaller than the typical single-domain size $(\sim 70 \mathrm{~nm})$ of the cobalt with spherical shape ${ }^{12}$, magnetic 
hysteresis stemming from irreversible magnetization and domain wall resonance can be excluded. Exchange resonance may be present in the present system, due to the fact that the size of FCC-Co/C nanocapsules is smaller than $100 \mathrm{~nm}$. If the magnetic loss only stems from the eddy current loss, then the values of $\mu^{\prime \prime}\left(\mu^{\prime}\right)^{-2} f^{-1}$ should be constant when the frequency is changed. We can call this the skin-effect criterion. As shown in Figure $3 \mathrm{~d}$, the values of $\mu^{\prime \prime}\left(\mu^{\prime}\right)^{-2} f^{-1}$ of the FCC-Co/C nanocapsules decrease remarkably with increasing frequency. Therefore, the magnetic loss in the present system may be caused mainly by the natural resonance and exchange resonance.

According to the transmission line theory, when a wave is normally incident to a absorber layer with a backed metal plate, the reflection loss (RL) curves at a given absorber thickness can be calculated from the complex permeability and permittivity by means of the following expressions ${ }^{8-11}$ :

$$
\begin{aligned}
& Z=Z_{\text {in }} / Z_{0}=\sqrt{\mu_{r} / \varepsilon_{r}} \tanh \left((j 2 \pi t / \lambda) \sqrt{\mu_{r} \varepsilon_{r}}\right) \\
& R L=20 \lg |(Z-1) /(Z+1)|
\end{aligned}
$$

Where $Z$ is the normalized input impedance related to the impedance in free space; $\lambda$ is the wavelength in free space; and $t$ the thickness of the absorber.

The three dimensional dependence of the RL of the $\mathrm{FCC}-\mathrm{Co} / \mathrm{C}$-paraffin composites with varying layer thickness (0.5-5.4 mm) on the EM wave frequency in the $2-18 \mathrm{GHz}$ range is presented in Figure 5. As shown in Figure 5, an optimal RL of $-33.4 \mathrm{~dB}$, corresponding to $99.95 \%$ absorption, is observed at $9.6 \mathrm{GHz}$ for the $2.6 \mathrm{~mm}$ thick layer. With increasing thickness of the absorption layer, the $\mathrm{RL}$ maximum of the FCC-Co/C nanocapsules shifts to lower frequency. When the thickness is thicker than the critical thickness, two peaks appear simultaneously. RL values exceeding $-20 \mathrm{~dB}$ in the $7.4-15 \mathrm{GHz}$ range are obtained by choosing an appropriate absorption-layer thickness between 1.7 and $3.3 \mathrm{~mm}$. This frequency range covers the absorption frequency range of the traditional sintered ferrites ${ }^{26,27}$. The thickness range is thinner than that of many earlier reported nanocomposites ${ }^{5,8-11}$. The good microwave absorbing properties of FCC-Co/C nanocapsules is considered to result from the excellent synergetic effect of the multi-dielectric relaxation loss and the multi-magnetic resonance loss.

The quarter-wavelength cancellation model has been successfully used to explain the relationship between RL peak frequency and absorber thickness for carbonyl-iron particle and $\mathrm{Ni} @ \mathrm{Ni}_{2} \mathrm{O}_{3}$ core-shell particles and $\mathrm{FeNi}_{3} / \mathrm{C}$ nanowires ${ }^{28-30}$. According to the model, the minimum RL can be gained at given frequencies if the thickness of the absorber $\left(t_{M}\right)$ satisfies:

$t_{m}=n c /\left(4 f_{m} \sqrt{\left|\varepsilon_{r} \mu_{r}\right|}\right)(\mathrm{n}=1,3,5 \ldots)$

Where $f_{M}$ is the peak frequency of RL, $\varepsilon_{r}$ and $\mu_{r}$ are the complex permittivity and permeability at $f_{M}$ and $c$ is the velocity of light.

Refer to Equation 2, the peak frequency is inversely proportional to the thickness. In addition, two RL peaks appear at a sufficiently large thickness. One at lower frequency is relative to the $\lambda / 4$ condition, and the other at higher frequency come from $3 \lambda / 4$ condition. We draw a comparison of the $t_{M}^{C A L}$ calculated through Equation 2 (n equals 1 and 3 ) with the $t_{M}^{S I M}$ simulated by Equation 1 for $\mathrm{FCC}-\mathrm{Co} / \mathrm{C}$-paraffin composite, as shown in Figure $6 \mathrm{a}$ and b. From Figure 6, the simulated results $t_{M}^{S I M}$ agree very well with the calculated values $t_{M}{ }^{C A L}$, which implies that the microwave absorption mechanism of FCC-Co/C-paraffin composite can be explained by the quarter-wavelength matching model $^{28}$.

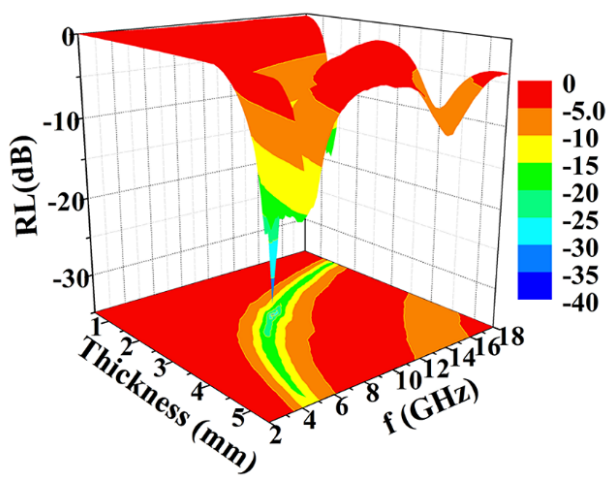

Figure 5. Three-dimensional representation of the RL derived from the measured and of the $\mathrm{FCC}-\mathrm{Co} / \mathrm{C}$ nanocapsules-paraffin composites as a function of the frequency.

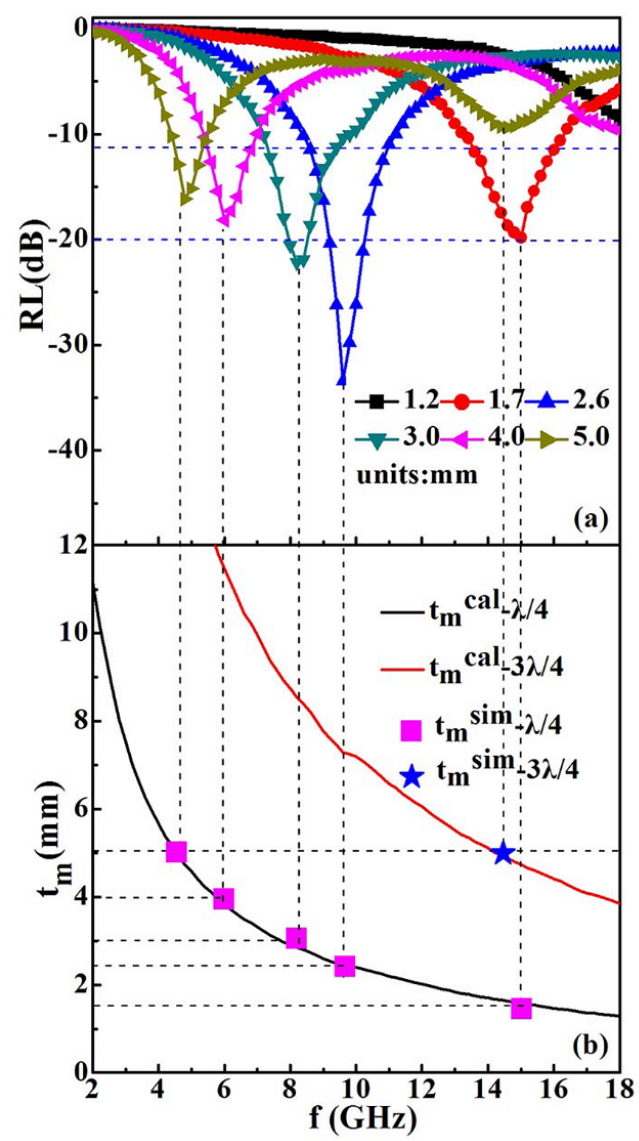

Figure 6. (a) Dependence of RL on frequency at various thicknesses for the $\mathrm{FCC}-\mathrm{Co} / \mathrm{C}$ nanocapsules-paraffin composites; and (b) Dependence of $\lambda / 4$ and $3 \lambda / 4$ thickness on frequency for the FCC-Co/C nanocapsules-paraffin composites. 


\section{Conclusion}

The FCC-Co/C core-shell structured nanocapsules have been synthesized by a facile hydrothermal and the main conclusions can be summarized as follows:

(1) The FCC-Co/C nanocapsules have a core-shell structure, with crystalline FCC-Co as the core and amorphous $\mathrm{C}$ as the shell. The size distribution is $5-20 \mathrm{~nm}$.

(2) The $T_{B}$ of FCC-Co/C nanocapsules is determined as $85 \mathrm{~K}$ from ZFC curve. The $M_{S}$ of FCC-Co/C nanocapsules at $300 \mathrm{~K}$ is $70.4 \mathrm{emu} / \mathrm{g}$. No coercive force exists in the hysteresis loop at $300 \mathrm{~K}$, indicating the FCC-Co/C nanocapsules are superparamagnetic.

(3) For FCC-Co/C nanocapsules-paraffin composite, an optimal RL of $-33.4 \mathrm{~dB}$, corresponding to 99.95\% absorption, is observed at $9.6 \mathrm{GHz}$ for the $2.6 \mathrm{~mm}$ thick layer. RL values exceeding $-20 \mathrm{~dB}$ in the $7.4-15 \mathrm{GHz}$ range are obtained by choosing an appropriate absorption-layer thickness between 1.7 and $3.3 \mathrm{~mm}$. The good microwave absorbing

\section{References}

1. Bregar VB. Advantages of ferromagnetic nanoparticle composite in microwave absorbers. Magnetics. IEEE Transactions on. 2004; 40(3):1679-1684.

2. Fujieda T, Ikeda S, Suzuki S, Abe T and Aono Y. Microwave absorption properties of $\mathrm{Fe}-\mathrm{SiO} 2$ nanocomposite powder. Journal of the Institute of Metals. 2002; 66:135-138.

3. Bhattacharya P, Hatui G, Mandal A, Das CK, Kumar R and Shami TC. Investigation of microwave absorption property of the core-shell structured $\mathrm{Li} 0.4 \mathrm{Mg} 0.6 \mathrm{Fe} 2 \mathrm{O} 4 / \mathrm{TiO} 2$ nanocomposite in X-band region. Journal of Alloys and Compounds. 2014; 590:331-340. http://dx.doi.org/10.1016/j.jallcom.2013.12.117.

4. Guan PF, Zhang XF and Guo J. Assembled Fe3O4 nanoparticles on graphene for enhanced electromagnetic wave losses. Applied Physics Letters. 2012; 101(15):153108. http://dx.doi. org/10.1063/1.4758931. PMid:22550357.

5. Liu XG, Geng DY, Ma S, Meng H, Tong M, Kang DJ, et al. Electromagnetic-wave absorption properties of $\mathrm{FeCo}$ nanocapsules and coral-like aggregates self-assembled by the nanocapsules. Journal of Applied Physics. 2008; 104(6):064319. http://dx.doi. org/10.1063/1.2982411.

6. Li G, Xie TS, Yang SL, Jin JH and Jiang JM. Microwave absorption enhancement of porous carbon fibers compared with carbon nanofibers. The Journal of Physical Chemistry C. 2012; 116(16):9196-9201. http://dx.doi.org/10.1021/jp300050u.

7. Liu XG, Ou ZQ, Geng DY, Han Z, Wang H, Li B, et al. Enhanced absorption bandwidth in carbon-coated supermalloy FeNiMo nanocapsules for a thin absorb thickness. Journal of Alloys and Compounds. 2010; 506(2):826-830. http://dx.doi.org/10.1016/j. jallcom.2010.07.085.

8. Liu XG, Geng DY, Meng H, Shang PJ and Zhang ZD. Microwaveabsorption properties of $\mathrm{ZnO}$-coated iron nanocapsules. Applied Physics Letters. 2008; 92(17):173117. http://dx.doi. org/10.1063/1.2919098.

9. Liu XG, Geng DY, Meng H, Li B, Zhang Q, Kang DJ, et al. Electromagnetic-wave-absorption properties of wire-like structures self-assembled by FeCo nanocapsules. Journal of properties of $\mathrm{FCC}-\mathrm{Co} / \mathrm{C}$ nanocapsules is considered to result from the excellent synergetic effect of the multi-dielectric relaxation loss and the multi-magnetic resonance loss.

(4) The microwave absorbing mechanism of FCC-Co/C nanocapsules-paraffin composite can be well explained by the quarter-wavelength cancellation model. Not only the peak frequency of RL but also the number of the peaks also have been determined by the quarter-wavelength cancellation model, which are demonstrated by the quantitative calculation.

\section{Acknowledgements}

This study has been supported partly by the National Natural Science Foundation of China (Grant No. 51201002), by the Research Grants Council of the HKSAR Government (PolyU 5236/12E), and by The Hong Kong Polytechnic University (G-YK59, 4-ZZ7L and G-YX3V).

Physics. D, Applied Physics. 2008; 41(17):175001. http://dx.doi. org/10.1088/0022-3727/41/17/175001.

10. Liu XG, Feng C, Or SW, Sun YP, Jin CG, Li WH, et al. Investigation on microwave absorption properties of $\mathrm{CuO} /$ $\mathrm{Cu} 2 \mathrm{O}$-coated $\mathrm{Ni}$ nanocapsules as wide-band microwave absorbers. RSC Advances. 2013; 3(34):14590-14594. http:/ dx.doi.org/10.1039/c3ra40937f.

11. Liu XG, Ou ZQ, Geng DY, Han Z, Xie ZG and Zhang ZD. Enhanced natural resonance and attenuation properties in superparamagnetic graphite-coated $\mathrm{FeNi} 3$ nanocapsules. Journal of Physics. D, Applied Physics. 2009; 42(15):155004. http:// dx.doi.org/10.1088/0022-3727/42/15/155004.

12. Ma S, Wang YB, Geng DY, Li J and Zhang ZD. Structure and magnetic properties of $\mathrm{Co}-\mathrm{Cr}$ solid-solution nanocapsules prepared by arc discharge. Journal of Applied Physics. 2005; 98(9):094304. http://dx.doi.org/10.1063/1.2125115.

13. Liu XG, Geng DY, Meng H, Cui WB, Yang F, Kang DJ, et al. Microwave absorption properties of FCC-Co/Al2O3 and FCCCo/Y2O3 nanocapsules. Solid State Communications. 2009; 149(1-2):64-67. http://dx.doi.org/10.1016/j.ssc.2008.10.015.

14. Jaumann T, Ibrhim EMM, Hampel S, Maier D, Leonhardt A and Büchner B. The synthesis of superparamagnetic cobalt nanoparticles encapsulated in carbon through high-pressure CVD. Chemical Vapor Deposition. 2013; 17(7-9):1-7.

15. Si PZ, Brück E, Zhang ZD, Tegus O, Buschow KHJ, Zhang WS, et al. Synthesis, structure and magnetic properties of Fe-Gd nanocapsules coated with $\mathrm{B} 2 \mathrm{O} 3 / \mathrm{H} 3 \mathrm{BO} 3$ and $\mathrm{Fe} 3 \mathrm{BO} 5+\mathrm{GdBO} 3$. Physica B, Condensed Matter. 2004; 353(1-2):1-8. http://dx.doi. org/10.1016/j.physb.2004.08.012.

16. Ibrahim EMM, Hamperl S, Kamsanipally R, Thomas J, Erdmann $\mathrm{K}$, Fuessel S, et al. Highly biocompatible superparamagnetic $\mathrm{Ni}$ nanoparticles dispersed in submicron-sized $\mathrm{C}$ spheres. Carbon. 2013; 63:358-366. http://dx.doi.org/10.1016/j.carbon.2013.06.091.

17. Ibrahim EMM, Hamperl S, Wolter AUB, Kath M, El-Gendy AA, Klingeler R, et al. Superparamagnetic FeCo and FeNi nanocomposites dispersed in submicrometer-sized $\mathrm{C}$ spheres. The Journal of Physical Chemistry C. 2012; 116(42):2250922517. http://dx.doi.org/10.1021/jp304236x. 
18. Ibrahim EMM, Hamperl S, Thomas J, Haase D, Wolter AUB, Khavrus VO, et al. Synthesis of superparamagnetic nanoparticles dispersed in spherically shaped carbon nanoballs. Journal of Nanoparticle Research. 2012; 14(9):1118. http://dx.doi. org/10.1007/s11051-012-1118-8.

19. Li YL, Zhao JZ, Su XD, Zhu Y, Wang Y, Tang L, et al. A facile aqueous phase synthesis of cobalt microspheres at room temperature. Colloid and Surfaces A: Physicochemcial and Engnineering Aspects. 2009; 336(1-3):41-45. http://dx.doi. org/10.1016/j.colsurfa.2008.11.012.

20. Wen SL, Liu Y, Zhao XC, Cheng JW and Li H. Synthesis, multinonlinear dielectric resonance and electromagnetic absorption properties of hep-cobalt particles. Journal of Magnetism and Magnetic Materials. 2014; 354:7-11. http://dx.doi.org/10.1016/j. jmmm.2013.10.030.

21. Wang ZH, Han Z, Geng DY and Zhang ZD. Synthesis, characterization and microwave absorption of carbon-coated $\mathrm{Sn}$ nanorods. Chemical Physics Letters. 2010; 489(4-6):187-190. http://dx.doi.org/10.1016/j.cplett.2010.02.056. PMid:21072139.

22. Muhammad E, Jamal A, Joy PA, Kurian P and Anantharaman MR. Synthesis of nickel-rubber nanocomposites and evaluation of their dielectric properties. Materials Science and Engineering B. 2009; 156(1-3):24-31. http://dx.doi.org/10.1016/j.mseb.2008.10.041.

23. Wang H, Dai YY, Gong WJ, Geng DY, Ma S, Li D, et al. Broadband microwave absorption of CoNi@C nanocapsules enhanced by dual dielectric relaxation and multiple magnetic resonances. Applied Physics Letters. 2013; 102(22):223113. http://dx.doi.org/10.1063/1.4809675.
24. Ma J, Li JG, Ni X, Zhang XD and Huang JJ. Microwave resonance in $\mathrm{Fe} / \mathrm{SiO} 2$ nanocomposites. Applied Physics Letters. 2009; 95(10):102505. http://dx.doi.org/10.1063/1.3224883.

25. Pan HS, Cheng XG, Zhang $\mathrm{CH}$, Gong $\mathrm{CH}$, Yu LG, Zhang JW, et al. Preparation of Fe2Ni2N/SiO2 nanocomposite via a two-step route and investigation of its electromagnetic properties. Applied Physics Letters. 2013; 102(1):012410. http://dx.doi. org/10.1063/1.4773991.

26. Yusoff AN, Abdullah MH, Ahmad SH, Jusoh SF, Manso AA and Hamid SAA. Electromagnetic and absorption properties of some microwave absorbers. Journal of Applied Physics. 2002; 92(2):876-882. http://dx.doi.org/10.1063/1.1489092.

27. Liu JR, Itoh M and Machida K. Electromagnetic wave absorption properties of $\alpha-\mathrm{Fe} / \mathrm{Fe} 3 \mathrm{~B} / \mathrm{Y} 2 \mathrm{O} 3$ nanocomposites in gigahertz range. Applied Physics Letters. 2003; 83(19):4017-4019. http:// dx.doi.org/10.1063/1.1623934.

28. Wang BC, Zhang JL, Wang T, Qiao L and Li FS. Synthesis and enhanced microwave absorption properties of $\mathrm{Ni@Ni2O3}$ core-shell particles. Journal of Alloys and Compounds. 2013; 567:21-25. http://dx.doi.org/10.1016/j.jallcom.2013.03.028.

29. Wang T, Han R, Tan GG, Wei JJ, Qiao L and Li FS. Reflection loss mechanism of single layer absorber for flake-shaped carbonyl-iron particle composite. Journal of Applied Physics. 2012; 112(10):104903. http://dx.doi.org/10.1063/1.4767365. PMid:23258940.

30. Sun YP, Liu XG, Feng C, Fan JC, Lv YH, Wang YR, et al. A facile synthesis of FeNi3@C nanowires for electromagnetic wave absorber. Journal of Alloys and Compounds. 2014; 586:688-692. http://dx.doi.org/10.1016/j.jallcom.2013.10.063. 